\title{
Feasibility of hydroxychloroquine adjuvant therapy in pregnant women with systemic lupus erythematosus.
}

\author{
En-Ling Liu ${ }^{1 *}$, Zheng Liu ${ }^{2 \#}$, Yu-Xiu Zhou ${ }^{3}$ \\ ${ }^{1}$ Department of Obstetrics and Gynecology, Tangshan Gongren Hospital, Tangshan, Hebei, PR China \\ ${ }^{2}$ Department of Rheumatism, Tianjin Medical University General Hospital, Tianjin, PR China \\ ${ }^{3}$ Department of Rheumatism, Tangshan Gongren Hospital, Tangshan, Hebei, PR China \\ \#These authors contributed equally to this paper
}

\begin{abstract}
Objective: This study aims to investigate the clinical effects of hydroxychloroquine adjuvant therapy in pregnant women with Systemic Lupus Erythematosus (SLE).

Methods: A total of 78 pregnant women with SLE from February 2016 to August 2017 were recruited and divided into two groups, control $(n=39)$ and observation $(n=39)$, by using a random number table. The observation group received hydroxychloroquine adjuvant therapy, whereas the control group received conventional symptomatic treatment. The lupus activity during pregnancy, term birth rate, pregnancy and fetus outcomes, new-born Apgar score, and neonatal weight of the two groups were compared.

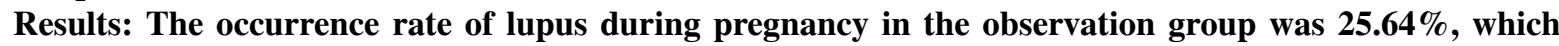
was significantly lower than that of the control group $(51.28 \%)(\mathbf{P}<0.05)$. The observation group achieved significantly higher term birth rate $(84.62 \%)$ than the control group $(58.97 \%)(P<0.05)$. The occurrence rates of bad pregnancy and fetus outcomes of the observation and control groups were $17.95 \%$ and $44.44 \%$, respectively, which showed a significant difference $(P<0.05)$. The new-born Apgar score and neonatal weight of the observation group were $9.61 \pm 0.25$ and $2.78 \pm 0.56 \mathrm{~kg}$, whereas those of the control group were $8.10 \pm 0.31$ and $2.47 \pm 0.58 \mathrm{~kg}$, showing a significant difference $(P<0.05)$.

Conclusions: Hydroxychloroquine adjuvant therapy exerts outstanding clinical effects to pregnant patients with SLE. This therapy not only improves pregnancy outcome but also reduces the occurrence of lupus. This method has a very high clinical application value and is worthy of further clinical research and applications.
\end{abstract}

Keywords: Pregnancy, SLE, Hydroxychloroquine, Clinical effect.

Accepted on November 30, 2017

\section{Introduction}

Systemic Lupus Erythematosus (SLE) is a common autoimmune disease with poorly understood pathogenesis [1]. SLE has diversified clinical manifestations and often implicates organ systems, such as skin, kidney, joints, and nervous centralis [2]. Moreover, this disease is often observed in women of child-bearing age. Pregnancy promotes disease development [3]. Delayed therapy to pregnant patients with SLE may cause abortion, pregnancy-induced hypertension syndrome, renal damage, and other complications. Therefore, positive exploration of effective therapy is necessary [4]. In this study, 78 pregnant patients with SLE from February 2016 to August 2017 were chosen to investigate the clinical effects of hydroxychloroquine adjuvant therapy.

\section{Information and Methods}

\section{General information}

A total of 78 pregnant patients with SLE from February 2016 to August 2017 were chosen and divided into two groups, control $(n=39)$ and observation $(n=39)$, by using a random number table. Patients who agreed with the diagnosis standards of SLEDE in immune epidemiology, were in the gestation period, and had complete clinical data were included. All patients volunteered to participate in this research program and signed the Informed Consent. This research agreed with the requirements of the Ethics Committee. Patients with complications of other serious diseases, drug allergy, mental disorder, communication barriers, and poor compliance to treatment were excluded. The control group aged from 22-39 y, with $(29.05 \pm 6.45)$ as the average. The course of disease was 
1-5 y, with $(2.92 \pm 1.21)$ as the average. The control group included six cases of lupus nephritis whose nephrosis was in non-activity period $(24 \mathrm{~h}$ urine protein $<0.5 \mathrm{~g}$ routine urinalysis). The observation group aged from 23-39 y, with $(29.11 \pm 6.22)$ as the average. The course of disease was $1-5 \mathrm{y}$, with $(2.82 \pm 1.41)$ as the average. The observation group included five cases of lupus nephritis whose nephrosis was in non-activity period $(24 \mathrm{~h}$ urine protein $<0.5 \mathrm{~g}$ routine urinalysis). A statistical analysis on the general information of age and course of disease of the two groups was carried out by SPSS 22.0. No significant difference was observed between the two groups $(\mathrm{P}>0.05)$.

\section{Methods}

The control group received conventional symptomatic treatment. Patients were asked to take metacotandracin (Tianjin Tianyao Pharmaceutical Co., Ltd, SFDA approval number: $2 \mathrm{H} 8910602,5 \mathrm{mg} / \mathrm{pc}$ ) orally twice per day, $5 \mathrm{mg}$ at a time. The observation group was provided with hydroxychloroquine adjuvant therapy. Patients were asked to take hydroxychloroquine (Shanghai Zhongxi Pharmaceutical Co., Ltd., SFDA approval number: H19990263, $100 \mathrm{mg} / \mathrm{pc}$ ) twice per day, $200 \mathrm{mg}$ at a time. Both groups were treated for $7 \mathrm{~d}$ successively.

\section{Observation indexes}

The lupus activity during pregnancy, term birth rate, pregnancy and fetus outcomes, new-born Apgar score, and neonatal weight of the two groups were compared. The score range of lupus activity during pregnancy was as follows: inactive, 0-4; slightly active, 5-9; moderately active, 10-14; and severely active, $\geq 15$. Furthermore, the conditions of pregnancy and fetus outcomes were premature rupture of fetal membranes, oligohydramnion, fetal distress, and restricted growth. The New-born Apgar score was full 10 score. Lastly, the score ranges for asphyxia manifestation were: severe asphyxia, 0-3; slight asphyxia, 4-7; and normal: $>7$.

\section{Statistical analysis}

A statistical analysis of the observation data of two groups was conducted by SPSS 22.0. Enumeration data (e.g., lupus activity during the pregnancy, term birth rate, and pregnancy and fetus outcomes) were expressed in percentages and examined by chisquare test. Measurement data (e.g., new-born Apgar score and neonatal weight) were expressed in mean \pm standard deviation and examined by t-test. $\mathrm{P}<0.05$ was considered to indicate statistical significance between the two groups.

\section{Results}

\section{Lupus activity during the pregnancy}

The occurrence rate of lupus during the pregnancy in the observation group was $25.64 \%$, which was significantly lower than that of the control group $(51.28 \%)(\mathrm{P}<0.05)$. Results are listed in Table 1.

Table 1. Comparison of lupus activity during the pregnancy between the two groups in percentages.

\begin{tabular}{|c|c|c|c|c|c|}
\hline Groups & Inactive & Slightly active & Moderately active & Severely active & Total occurrence rate \\
\hline Observation & $29(74.36)$ & $6(15.38)$ & $4(10.26)$ & $0(0.0)$ & $10(25.64)$ \\
\hline Control & $19(48.72)$ & $10(25.64)$ & $8(20.51)$ & $2(5.13)$ & $20(51.28)$ \\
\hline$x^{2}$ & & & & & 5.4166 \\
\hline$P$ & & & & & 0.0199 \\
\hline
\end{tabular}

\section{Term birth rate}

The observation group achieved significantly higher term birth rate $(84.62 \%)$ than the control group $(58.97 \%)(\mathrm{P}<0.05)$. Results are listed in Table 2.

Table 2. Comparison of term birth rate between the two groups in percentages.

\begin{tabular}{lll}
\hline Groups & Premature birth & Term birth \\
\hline Observation $(n=39)$ & $6(15.38)$ & $33(84.62)$ \\
\hline
\end{tabular}

\begin{tabular}{lll}
\hline Control $(n=39)$ & $16(41.03)$ & $23(58.97)$ \\
\hline$X^{2}$ & 6.3311 \\
\hline$P$ & 0.0118 \\
\hline
\end{tabular}

\section{Pregnancy and fetus outcomes}

The occurrence rates of bad pregnancy and fetus outcomes of the observation and control groups were $17.95 \%$ and $44.44 \%$, respectively, showing a significant difference $(\mathrm{P}<0.05)$. Results are listed in Table 3.

Table 3. Comparison of pregnancy and fetus outcomes between the two groups in percentages.

\begin{tabular}{llll}
\hline Groups & $\begin{array}{l}\text { Premature rupture of fetal Oligohydramnion } \\
\text { membranes }\end{array}$ & Fetal distress Restricted growth Total rate \\
\hline
\end{tabular}




\begin{tabular}{llllll}
\hline Observation $(\mathrm{n}=39)$ & $2(5.13)$ & $2(5.13)$ & $2(5.13)$ & $1(2.56)$ & $7(17.95)$ \\
\hline Control $(\mathrm{n}=39)$ & $6(15.38)$ & $4(10.26)$ & $4(10.26)$ & $3(7.69)$ & $17(44.44)$ \\
\hline$X^{2}$ & & & & 6.0185 \\
\hline$P$ & & & 0.0141 \\
\hline
\end{tabular}

\section{New-born Apgar score and neonatal weight}

Significant differences were observed between the control and observation groups in terms of new-born Apgar score and neonatal weight $(\mathrm{P}<0.05)$. Results are shown in Table 4 .

Table 4. Comparison of new-born Apgar score and neonatal weight between the two groups.

\begin{tabular}{lll}
\hline Groups & $\begin{array}{l}\text { New-born Apgar score } \\
\text { (scores) }\end{array}$ & Neonatal weight $\mathbf{( k g )}$ \\
\hline Observation $(\mathrm{n}=39)$ & $9.61 \pm 0.25$ & $2.78 \pm 0.56$ \\
\hline Control $(\mathrm{n}=39)$ & $8.10 \pm 0.31$ & $2.47 \pm 0.58$ \\
\hline $\mathrm{t}$ & 23.6786 & 2.4012 \\
\hline $\mathrm{P}$ & 0.0000 & 0.0187 \\
\hline
\end{tabular}

\section{Discussion}

SLE is an autoimmune disease caused by combined action of multiple factors. This condition is often observed in women of child-bearing age [5]. According to relevant literature, pregnancy and delivery are the causes of SLE deterioration. Some scholars believed that about $50 \%$ of pregnant patients with SLE suffer from exacerbation and experience poor pregnancy and fetus outcomes [6]. This finding is mainly related to changes in hormonal levels during pregnancy. During pregnancy, women have relatively higher estrogen levels, which induce activation of polyclone B cells, promote expression of autoantibodies, and enhance autoimmune responsibility. Therefore, lupus occurs and further develops [7].

In treatment to pregnant patients with SLE, metacortandracin is the first choice of medication. Metacortandracin is a glucocorticoid drug that is often used in small-dose maintenance treatment. Large-dose treatment can be chosen upon aggravation depending on the actual situations of patients. Relevant research demonstrated that treatment with large-dose hormone drugs during pregnancy may cause pregnancy-induced hypertension syndrome, premature rupture of fetal membrane, and diabetes [8]. Such after effects not only threaten the life safety of patients but also affect the fetus.

Application of hydroxychloroquine, a 4-amino quinolone, during SLE treatment achieves good clinical effect. According to abundant research, hydroxychloroquine treatment in pregnant women with SLE mainly includes the following points: Anti-inflammatory action relieves inflammation by inhibiting the chemotaxis of polymorphonuclear cells [9]. Meanwhile, this response can intercept the biosynthesis of prostate and has very high clinical value. Immunosuppressive action can inhibit lymphocyte reactions effectively and cause lymphocyte apoptosis, thus reducing the production of IL-2, IL-6, IL-1A, IL-1B, and TNF- $\alpha$. Moreover, this action inhibits the processing and growth of autoantigens, thus inhibiting autoimmune responses. In addition, hydroxychloroquine can be combined with hormone drugs, thereby reducing usage of hormone drugs and decreasing occurrences of untoward effects [10].

This study concluded that the occurrence rate of lupus during pregnancy in the observation group was $25.64 \%$, which was significantly lower than that of the control group $(51.28 \%)$ $(\mathrm{P}<0.05)$. The observation group achieved significantly higher term birth rate $(84.62 \%)$ than the control group $(58.97 \%)$ $(\mathrm{P}<0.05)$. The occurrence rates of bad pregnancy and fetus outcomes of the observation and control groups were $17.95 \%$ and 44.44\%, respectively, which showed a significant difference $(\mathrm{P}<0.05)$. The New-born Apgar score and neonatal weight of the observation group were $9.61 \pm 0.25$ and $2.78 \pm$ $0.56 \mathrm{~kg}$, whereas those of the control group were $8.10 \pm 0.31$ and $2.47 \pm 0.58 \mathrm{~kg}$, which also demonstrated a significant difference $(\mathrm{P}<0.05)$. These findings basically conform to relevant literature reports. Therefore, hydroxychloroquine adjuvant therapy has higher clinical effects in pregnant patients with SLE than the conventional treatment. This therapy produced a synergistic effect with hormone drugs and further stabilized the disease conditions of patients, thus improving the prognosis of patients and reducing the occurrences of poor pregnancy outcomes. Meanwhile, this medical treatment can protect the safety of new-borns and entails a kind of therapeutic drug that deserves clinical applications and popularization.

\section{Conclusion}

Overall, hydroxychloroquine adjuvant therapy exerts an outstanding clinical effect on pregnant patients with SLE. This therapy can not only improve pregnancy outcomes but also reduce the occurrence of lupus. Moreover, this therapy has very high clinical application value and is worthy of further clinical research and applications.

\section{Acknowledgments}

This work was supported by grants from the Science and Technology Department of Hebei Province (162777190). 


\section{References}

1. Magro-Checa C, Zirkzee EJ, Huizinga TW, Steup-Beekman GM. Management of neuropsychiatric systemic lupus erythematosus: current approaches and future perspectives. Drugs 2016; 76: 459-483.

2. Zhang D, Gong J. Clinical study on the level of glucose metabolism and placental $\mathrm{NO}$ in patients with gestational hypertension. Biomed Res India 2017; 28: 3340-3343.

3. Crampton SP, Morawski PA, BollandS. Linking susceptibility genes and pathogenesis mechanisms using mouse models of systemic lupus erythematosus. Dis Model Mech 2014; 7: 1033-1046.

4. Hao DF, Feng G, Chu WL, Chen ZQ, Li SY. Role of topical and systemic therapy in patients with diabetic foot ulcer. Lat Am J Pharm 2016; 35: 105-109.

5. Liu CC, Kao AH, Manzi S, Ahearn JM. Biomarkers in systemic lupus erythematosus: challenges and prospects for the future. Ther Adv Musculoskelet Dis 2013; 5: 210-233.

6. Unlu O, Zuily S, Erkan D. The clinical significance of antiphospholipid antibodies in systemic lupus erythematosus. Eur J Rheumatol 2016; 3: 75-84.

7. Min A. A case report of postpartum hemolytic uremic syndrome. Biomed Res India 2017; 28: 3808-3810.
8. Lateef A, Petri M. Unmet medical needs in systemic lupus erythematosus. Arthritis Res Ther 2012; 14: 4.

9. Cai X, Wang Z, Wang C, Ni J, Yao J, Cao J, Xu Y, Zhang L, Yang G. Biodistribution and pharmacokinetics of cationic liposomes containing sophoridine following systemic administration in pancreatic tumor-bearing mice. Lat Am J Pharm 2016; 35: 1077-1082.

10. Barnard RA, Wittenburg LA, Amaravadi RK, Gustafson DL, Thorburn A, Thamm DH. Phase I clinical trial and pharmacodynamic evaluation of combination hydroxychloroquine and doxorubicin treatment in pet dogs treated for spontaneously occurring lymphoma. Autophagy 2014; 10: 1415-1425.

\section{*Correspondence to}

En-Ling Liu

Department of Obstetrics and Gynecology

Tangshan Gongren Hospital

PR China 